\title{
Ca-asp bound X-ray structure and inhibition of Bacillus anthracis dihydroorotase (DHOase)
}

Amy J. Rice, Hao Lei, Bernard D. Santarsiero, Hyun Lee*, Michael E. Johnson*

${ }^{1}$ Center for Biomolecular Sciences and Department of Medicinal Chemistry and Pharmacognosy, University of Illinois at Chicago, 900 S. Ashland, IL 60607, USA

*To whom correspondence should be addressed.

Michael E. Johnson: (Phone) 312-996-9114, (Fax) 312-413-9303, (Email) mjohnson@uic.edu Hyun Lee: (Phone) 312-413-9304, (Fax) 312-413-9303, (E-mail) danielhl@uic.edu 


\section{Introduction}

The pyrimidine biosynthesis pathway is an attractive target for antibacterial drug design, as cells rely on nucleotide synthesis for survival and proliferation, and pyrimidines are essential building blocks of RNA and DNA. Mammals have a large, multifunctional dihydroorotate synthetase (CAD) enzyme for the first three steps of the de novo pyrimidine biosynthesis pathway, while prokaryotes use three separate monofunctional enzymes for each. ${ }^{1,2}$ Dihydroorotase (DHOase), the third enzyme in the de novo pathway, is responsible for the reversible cyclization of carbamyl-asparate (Ca-asp) to dihydroorotate (DHO) (Fig. 1A). Historically, DHOase was divided into two evolutionary classes, with very low sequence identity $(<30 \%)$ between classes. Class I DHOases are found in gram-positive bacteria, mold, and insects, while Class II DHOases are found in gram-negative bacteria and fungi. There are two distinct differences between these two classes of DHOases. First, the main differences are structural, with Class II being slightly smaller at $\sim 38 \mathrm{kDa}$, and with a long flexible catalytic loop, compared to $\sim 45 \mathrm{kDa}$ Class I enzymes with either no or a very short corresponding loop. Second, DHOases contain four conserved histidines and one aspartate that coordinate the active site $\mathrm{Zn}^{2+}$ ions. Two active site $\mathrm{Zn}^{2+}$ ions in Class I are bridged by an aspartate, whereas a carboxylated lysine serves the same role in Class II. More recently, a third class has been suggested for human and Porphyromonas gingivalis DHOases because they have higher sequence similarity to Class I, but have a long catalytic loop and the active site $\mathrm{Zn}^{2+}$ ions are bridged by a carboxylated lysine, distinctive to Class II (Fig. 1B). ${ }^{3}$

So far, the Class II DHOase from Escherichia coli has been most extensively studied, producing many apo and complex structures. The structure revealed DHOase to be a homodimer zinc metalloenzyme that belongs to the 'amidohydrolase superfamily', a classification proposed 
for a group of functionally diverse metal-dependent hydrolase enzymes with a central $(\beta / \alpha)_{8}$ barrel fold in the catalytic domain. ${ }^{4,5}$ The mechanism of DHOase was structurally determined as well, and is a cyclization reaction where the Ca-asp is stabilized by the active site $\mathrm{Zn}^{2+}$ and several active site residues (Asn44, Arg20, His254), while the carboxylate of Asp250 abstracts a proton from the amide nitrogen of Ca-asp, allowing for a nucleophilic attack (Fig. 1C). ${ }^{2}$ The proposed chemical mechanism coincides with previously observed effects of $\mathrm{pH}$ on DHOase activity, where the forward enzymatic reaction is favored at lower $\mathrm{pH}$, while the reverse reaction is favored at higher $\mathrm{pH} .^{6,7}$

Crystal structures of ligand-bound DHOase show that the catalytic loop extends toward the active site when Ca-asp is bound, whereas it is in an 'out' formation when DHO is the substrate. ${ }^{8-10}$ The ligand and inhibitor complexes of E. coli DHOase and mutagenesis studies identified the importance of two threonine residues (T109, T110) on the flexible loop of Class II DHOase to stabilize the transition-state of Ca-asp, with T110 mutations resulting in decreased activity and T109 mutations demonstrating the essentiality of the hydrogen bond interaction from the hydroxyl group for converting Ca-asp to DHO (Fig. 1C). ${ }^{8-12}$ However, Class I DHOase has no distinct loop, and there are no structures of substrate or inhibitor bound Class I enzymes available to date to elucidate the interactions that would replace the role of these important threonines.

Currently, no inhibitors for gram-positive bacterial DHOase have been identified. Bacillus anthracis is the Gram-positive etiologic agent of anthrax, and DHOase has been identified as critical for its survival in human serum. ${ }^{13}$ Human anthrax infections can be developed through exposure via skin, ingestion of infected animals, inhalation of spores, or more recently, by injection. ${ }^{14}$ Early diagnosis and treatment is critical, but can be difficult if the 
person is unaware of being exposed to the pathogen as the initial clinical presentation is consistent with flu-like symptoms. ${ }^{15}$ Even with treatment, the mortality rates of gastrointestinal and inhalation anthrax are $>40 \% .{ }^{16,17}$ Current treatment is focused on post-exposure prophylaxis (PEP), using a combination of antibiotics and antitoxins. Unfortunately, the long term therapy recommended with antimicrobials, such as $\beta$-lactams, introduce the risk of developing resistance. Therefore, developing novel antibiotics against $B$. anthracis would increase treatment options and make anthrax bioterrorism attacks less feasible.

Here, we present the first crystal structure of substrate-bound B. anthracis DHOase, which provides further insight into the differences in the catalytic loop between Class I and Class II DHOase and the role it may play in inhibitor or substrate binding and recognition. We previously screened inhibitors of Class II DHOase and DHO analogs against B. anthracis DHOase, and observed little to no inhibition. ${ }^{18}$ To identify potential inhibitors, we screened 28,000 compounds and fragments against $B$. anthracis DHOase using a high throughput enzymatic colorimetric assay ${ }^{18,19}$ and a thermal shift binding assay ${ }^{20-23}$, followed by an "orthogonal" surface plasmon resonance analysis. ${ }^{24}$

A

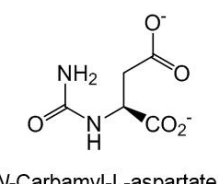

$N$-Carbamyl-L-aspartate (Ca-asp)

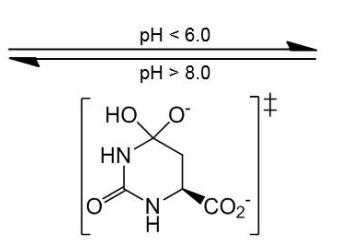

然

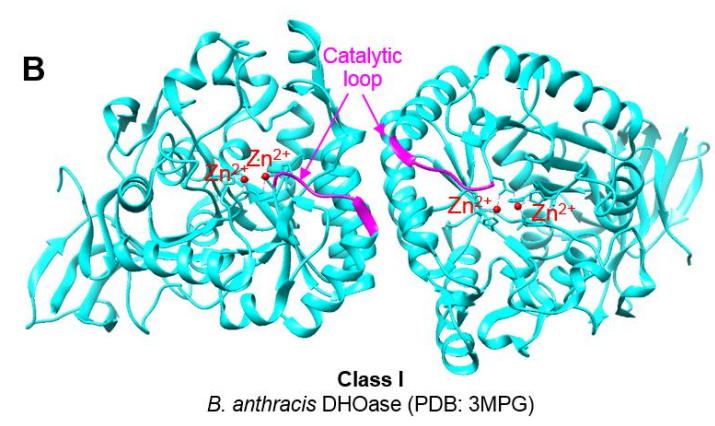

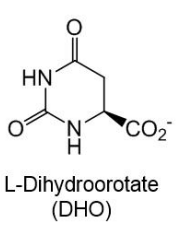

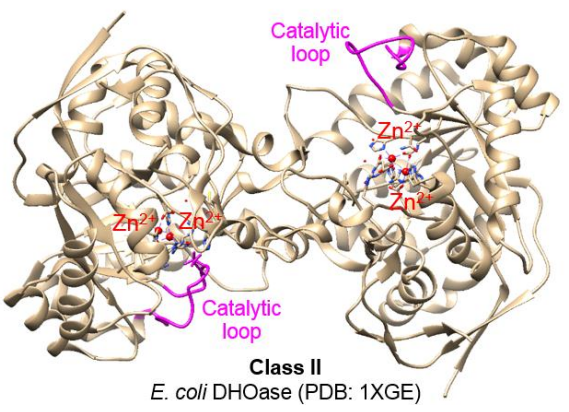

C

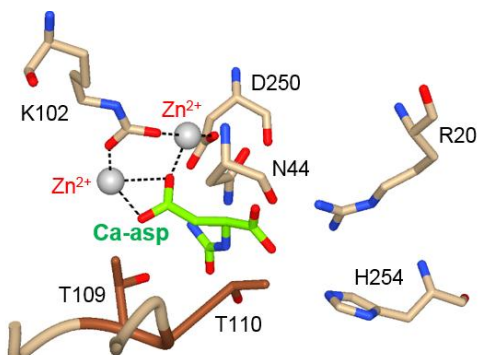

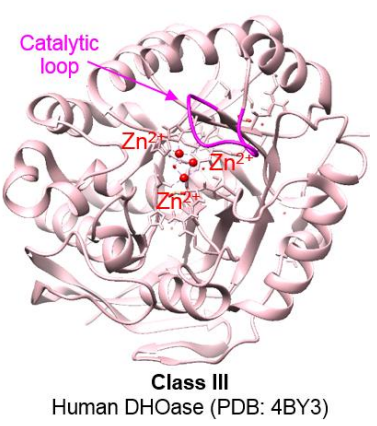


Figure 1. The mechanism of DHOase. (A) The enzyme catalyzes the reversible cyclization of Ca-asp to DHO. (B) Ca-asp is stabilized by the $\mathrm{Zn}^{2+}$ ions coordinated by a carboxylated $\mathrm{K} 102$, while D250 abstracts the proton from the amide nitrogen of Ca-asp. (PDB: 1XGE). The exocyclic carboxyl group of Ca-asp is stabilized by N44, R20, and H254, in addition to the T109 and T110 from the 'in' formation of the catalytic loop. (C) Comparison of the three classes of DHOase with the catalytic loop highlighted in pink.

\section{Results and discussion}

\subsection{Structure of substrate-bound DHOase from $B$. anthracis}

Class II DHOase from $E$. coli has been extensively studied with many reported crystal structures of apo wild-type and mutants and complexes with substrates or inhibitors. ${ }^{8-10,12}$ However, only apo structures have been solved for the two Gram positive bacteria DHOases from B. anthracis and Staphylococcus aureus. Here, we report the first substrate-bound crystal structure of a bacterial Class I DHOase. (PDB: 4YIW) The Ca-asp bound B. anthracis DHOase structure was solved and deposited as a homodimer at $2.45 \AA$, with chains A and B in one asymmetric unit. The substrate, Ca-asp, was found in the active site of each monomer, and both substrates have relatively weak electron density due to low substrate occupancy (Fig. 2A). The data processing and structure refinement statistics are summarized in Table 1. The overall structure of $B$. anthracis DHOase in complex with the Ca-asp looks similar to the apo structure (PDB: 3MPG) with an overall RMSD of $0.359 \AA$, indicating that binding of the Ca-asp did not cause much structural change. An overlay of Ca-asp-bound E. coli DHOase (PDB:1XGE) and our $B$. anthracis DHOase complex structure reveal a nice overlap in the catalytic aspartic acid (D250 and D304 in Fig. 2B). Another aspartate (D151) of B. anthracis DHOase aligns well with the 
carboxylated lysine (K102) of E. coli DHOase, serving the same purpose of bridging two active site $\mathrm{Zn}^{2+}$ ions. These two $\mathrm{Zn}^{2+}$ ions hold the substrate Ca-asp in position along with the active site residues, Arg60, Asn93, and His308, which also overlap well, suggesting the substrate is stabilized by the same hydrogen bond interactions. However, a major structural difference between the Class I and Class II DHOase is the longer catalytic loop of the Class II counterparts (shown in brown color in Fig. 2C). In the case of E. coli DHOase, previous studies have shown that the hydrogen bond interaction between Ca-asp and the two threonines (T109 and T110) on the catalytic loop play a crucial role. ${ }^{10}$ However, neither of the threonine residues is present in $B$. anthracis DHOase, so it has been previously hypothesized that the glycine (G152) in the shorter Class I loop may serve this function. ${ }^{3}$ According to our complex structure, this could be the case since the peptide backbone hydrogen of glycine is located at a distance of $3.75 \AA$ from Ca-asp, which is within weak H-bond interaction range. (shown in blue dotted line in Fig. 2C). Flexible catalytic loops from the three DHOase classes are aligned for comparison (Fig. 2D and Fig. 2E). In the case of both Class II and III, long catalytic loops showed a distinct shift (dotted arrows) in the substrate-bound structures, indicating the regulatory role of the loops. On the other hand, the corresponding loop of B. anthracis DHOase (Class I) is very short and remained in the same location in the presence and absence of the substrate Ca-asp, and whether this loop plays the same stabilizing role or not is yet to be determined. 

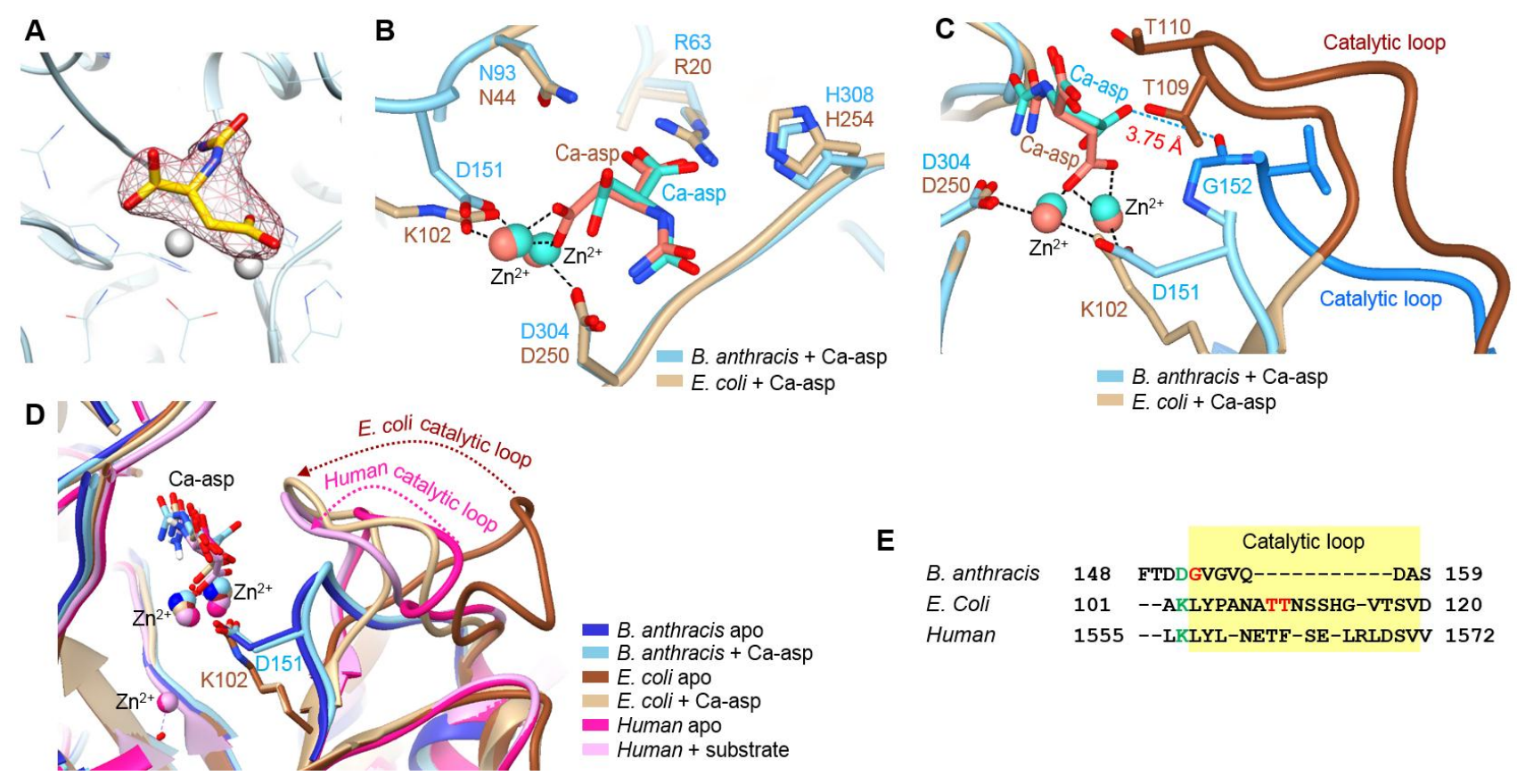

E

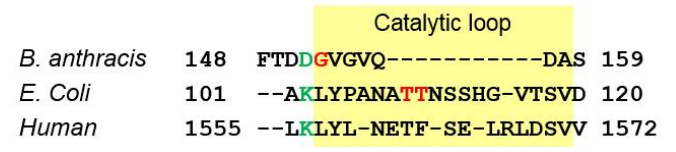

Figure 2. Comparison of Class I and Class II DHOase. (A) Electron density of Ca-asp in the active site. The ligand density is contoured at $2 F_{o}-F_{c}=0.51 \mathrm{rmsd}$. (B) Overlay of substrate bound B. anthracis DHOase (PDB:4YIW, cyan) and E. coli DHOase (PDB:1XGE, tan). (C) Catalytic loop of E. coli DHOase (PDB:1XGE) highlighted in blue, and H-bond interaction of substrate with the peptide backbone of glycine in $B$. anthracis DHOase. (PDB:4YIW). (D) Overlay of the catalytic loop. (B. anthracis apo PDB:3MPG; B. anthracis + Ca-asp PDB:4YIW; E. coli apo PDB:2EG6; E. coli + Ca-asp PDB:1XGE; human apo PDB:4BYE; human + Ca-asp PDB:4C6I). (E) Sequence alignment of catalytic loop highlighted in yellow.

Table 1. X-ray crystallography statistics for the refinement of 4YIW.

\begin{tabular}{ll}
\hline \multicolumn{2}{l}{ 4YIW } \\
\hline Data collection & $\mathrm{P} 2(1)($ No. 4) \\
Space group & $50.18 \AA 81.68 \AA 104.53 \AA 100.29^{\circ}$ \\
Cell constants $(\mathrm{a}, \mathrm{b}, \mathrm{c}, \beta)$ & $19.72(2.45) \AA$ \\
Resolution $(\AA)$ & 211917 \\
Total No. of reflections & \\
\hline
\end{tabular}




\begin{tabular}{ll}
\hline No. of averaged reflections (unique reflections) & 32367 \\
Redundancy & 6.5 \\
$R_{\text {merge }}(\%)$ & $14.0(99.1)^{\dagger}$ \\
$<I / \sigma(I)>$ & $5.84(1.08)^{\dagger}$ \\
Completeness $(\%)$ & $71.0(99.6)^{\dagger}$ \\
Refinement & \\
Resolution range $(\AA)$ & $19.72-2.45$ \\
No. of reflections & 28978 \\
No. of reflections in test set & 1521 \\
Completeness $(\%)$ & 99.33 \\
$R_{\text {cryst }}(\%)$ & 20.6 \\
$R_{\text {free }}(\%)$ & 26.5 \\
Wilson $B$ factor $\left(\AA^{2}\right)$ & 36.9 \\
Mean B factor & 42.0 \\
No. of protein molecules in asymmetric unit & 2 \\
R.m.s.d.s from ideal geometry & \\
Bond lengths $(\AA)$ & 0.006 \\
Bond angles $\left({ }^{\circ}\right)$ & 1.023 \\
Ramachandran plot & \\
Favoured $(\%)$ & 93.85 \\
Allowed $(\%)$ & 5.56 \\
Disallowed $(\%)$ & 0.59 \\
No. of solvent molecules & 310 \\
Ligand & $\mathrm{Zn}$ \\
& $\mathrm{NCD}$ \\
Mean B factor NCD & 67.9 \\
Mean B factor $\mathrm{H}_{2} \mathrm{O}$ & 34.1 \\
\hline
\end{tabular}

${ }^{\dagger}$ Highest resolution shell shown in parentheses

\subsection{Mutagenesis Studies}

Based on our substrate bound crystal structure, three active site residues (Arg63, Asn93, and Asp304) appear to stabilize the substrate and $\mathrm{Zn}^{2+}$ ion, indicating that these three residues could affect enzyme activity of $B$. anthracis DHOase. To further elucidate substrate-enzyme interactions, the three residues were sequentially mutated to an alanine. The affinities $\left(\mathrm{K}_{\mathrm{M}}\right)$ of wild-type B. anthracis DHOase for Ca-asp and DHO are essentially identical, at $112 \pm 24 \mu \mathrm{M}$ and $114 \pm 15 \mu \mathrm{M}$, respectively (Table 2). In contrast, the affinities of E. coli DHOase for Ca- 
asp and DHO differ significantly, with $\mathrm{K}_{\mathrm{M}}$ values of $1700 \mu \mathrm{M}$ and $80 \mu \mathrm{M}$, respectively. ${ }^{2}$ The catalytic activities of $B$. anthracis DHOase and E. coli DHOase also differ. For instance, the $E$. coli DHOase $\mathrm{k}_{\mathrm{cat}}$ values for Ca-asp and DHO are $160 \pm 8 \mathrm{~s}^{-1}$ and $100 \pm 1.6 \mathrm{~s}^{-1}$, respectively, compared to $2.1 \pm 0.1 \mathrm{~s}^{-1}$ and $1.9 \pm 0.1 \mathrm{~s}^{-1}$ for $B$. anthracis DHOase. This finding is similar to the catalytic kinetics of S. aureus DHOase, another active Class I DHOase. ${ }^{25}$ Interestingly, the catalytic efficiency $\left(\mathrm{k}_{\mathrm{cat}} / \mathrm{K}_{\mathrm{M}}\right)$ of B. anthracis DHOase with Ca-asp as the substrate is similar to that of E. coli DHOase when Thr110 is mutated to Serine, and the essential Thr109 is maintained. The serine could still make the hydrogen bond interaction between the loop and Caasp, but this mutation caused a 6-fold decrease in $\mathrm{k}_{\mathrm{cat}} / \mathrm{K}_{\mathrm{M}}$ compared to the wild-type, making its activity comparable to that of B. anthracis and S. aureus DHOases. Previously, it has been hypothesized that either the glycine in the shorter loop of Class I DHOase or the ATCase domain plays the critical role of the threonines in Class I DHOase, explaining why some Class I DHOase are active, while others, such as A. aeolicus DHOase is inactive unless it associates with the ATCase domain. The comparison of our kinetic parameters suggests that although the peptide backbone of glycine in Class I DHOase may contribute H-bond interactions for enzyme activity, an association with the ATCase domain may increase the enzyme activity. Despite the $\sim 6$-fold difference in $\mathrm{k}_{\mathrm{cat}} \mathrm{K}_{\mathrm{M}}$ values, the crystal structures show that Ca-asp binds in a similar manner in both classes of DHOase and has the same physicochemical interactions with the active site residues.

The E. coli DHOase Asp250 stabilizes a $\mathrm{Zn}^{2+}$ ion and initiates the enzyme reaction, and this aspartic acid residue is required for catalytic activity. This is also the case for Class I $B$. anthracis DHOase, as mutagenesis of D304 to alanine completely abolished activity in $B$. anthracis DHOase, with minimal recovery of activity after supplementing with zinc sulfate up to 
$2.5 \mathrm{mM}$. The R63A mutant also showed no enzyme activity with either DHO or Ca-asp. This is because Arg63 stabilizes the exocyclic carboxylic acid of both the substrate and product in the active site. On the other hand, Asn93 has an electrostatic interaction with the carboxylic group of Ca-asp, which undergoes nucleophilic attack by the amide nitrogen to form DHO. The N93A mutant has minor activity, even with almost 10-fold higher concentrations of Ca-asp compared to wild-type DHOase, most likely due to the inability to stabilize Ca-asp in the active site. When DHO is the substrate, no enzyme activity is observed, presumably because DHO lacks the carboxylic terminal to interact with Asn93 (Table 2).

Table 2. Comparison of kinetic values of wild-type and mutants of B. anthracis DHOases.

\begin{tabular}{ccccc}
\hline Enzyme & Substrate & $\mathrm{K}_{\mathrm{M}}(\mu \mathrm{M})$ & $k_{\text {cat }}\left(\mathrm{s}^{-1}\right)$ & $K_{\text {cat }} / K_{M}\left(\mathrm{M}^{-1} \mathrm{~s}^{-1}\right)$ \\
\hline WT-DHOase & Ca-asp & $112 \pm 24$ & $2.1 \pm 0.1$ & $1.9 \times 10^{4}$ \\
WT-DHOase & DHO & $114 \pm 16$ & $1.9 \pm 0.1$ & $1.7 \times 10^{4}$ \\
R63A-DHOase & Ca-asp & na & na & na \\
R63A-DHOase & DHO & na & na & na \\
N93A-DHOase & Ca-asp & $1183 \pm 251$ & $1.4 \pm 0.1$ & $1.2 \times 10^{3}$ \\
N93A-DHOase & DHO & na & na & na \\
D304A-DHOase & Ca-asp & na & na & na \\
D304A-DHOase & DHO & na & na & na \\
\hline
\end{tabular}

na : no activity (not measurable)

\subsection{High-throughput Screening and hit validation}

Previously, inhibitors of E. coli and P. falciparum DHOase, such as 5-fluoroorotate (FOA), 5aminoorotate (AOA), and three analogs of DHO showed no inhibition of $B$. anthracis DHOase. ${ }^{18}$ Therefore, to identify inhibitors for $B$. anthracis DHOase, a high-throughput screen (HTS) of 3,352 fragments from the Chembridge fragment and Zenobia fragment libraries were performed using a colorimetric enzymatic assay. A second thermal shift binding assay was performed to identify those that bind to the target enzyme. The enzymatic colorimetric assay uses a DAMO- 
TSC color mix to detect the ureido moiety of Ca-asp, as previously described. ${ }^{18}$ Compounds that inhibit DHOase should prevent the reverse cyclization process from DHO to Ca-asp. Therefore, absence or lowered concentrations of Ca-asp will exhibit little to no color change. For our studies, any fragment that showed $<50 \%$ of color development (red square in Fig. 3A) compared to the control suggested enzyme activity inhibition and were considered to be initial hits. HTS using this method resulted in 142 hits. The thermal shift binding assay uses a fluorescence dye to monitor unfolding and exposure of hydrophobic regions of an enzyme while gradually increasing the temperature. ${ }^{26}$ If a fragment binds to DHOase, it should stabilize the enzyme, resulting in a shift in melting temperature ( $\mathrm{T}_{\mathrm{m}}$ ) to a higher temperature (Fig. 3C). Fragments that showed $>2{ }^{\circ} \mathrm{C}$ shift in temperature were identified as hits (Fig. 3B). This second method of HTS resulted in 160 hits, with 10 fragments overlapping with the enzymatic screen. One of the disadvantages of the fluorescence thermal shift assay is that fragments which saturate the target may not show any temperature shift by displaying a broad fluorescence curve. For that reason, we did not want to disregard any promising hits from our enzymatic assay. After testing the enzymatic hits for compound interference on color development, we selected 14 fragments to perform a secondary confirmation assay. Surface plasmon resonance (SPR) was used to eliminate non-specific binders and to determine the binding affinity (dissociation equilibrium constant, $\mathrm{K}_{\mathrm{D}}$ ). Promiscuous binders were identified as those that did not show a trend toward a saturable curve (Fig. S5). Sensorgrams of compound $\mathbf{4}$ are shown at eight different concentrations in Fig. 3D as an example, and the determined $K_{D}$ values of 8 final fragment hits varied between $67 \mu \mathrm{M}$ and $334 \mu \mathrm{M}$ (Fig. 4). 
A

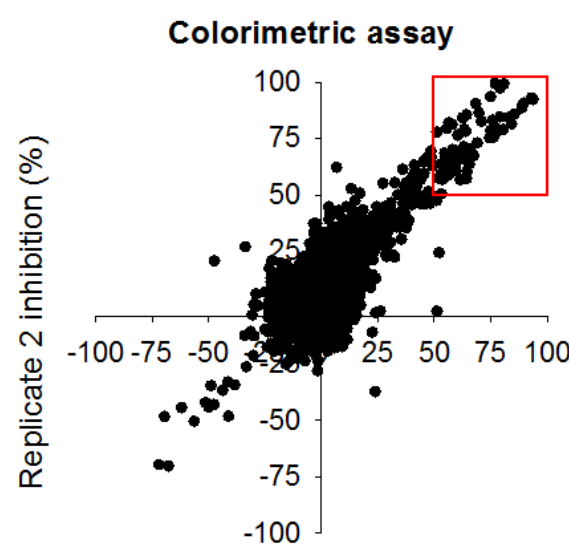

Replicate 1 inhibition (\%)

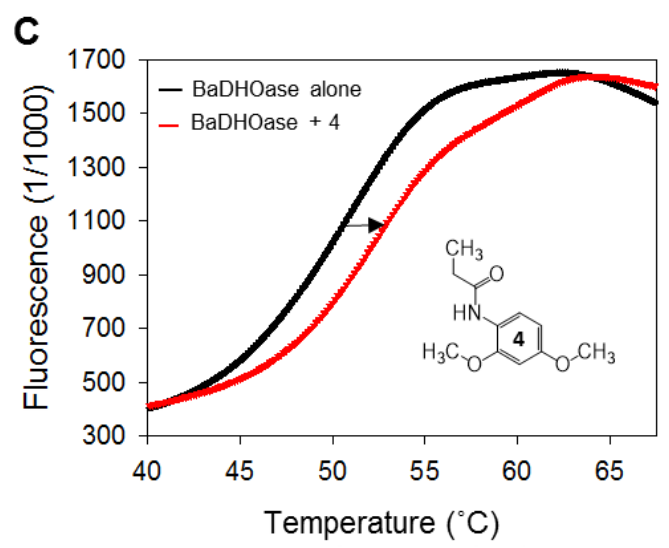

B

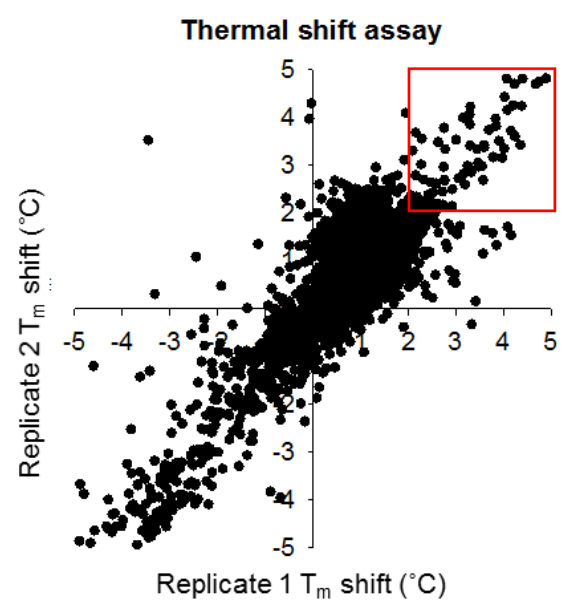

D

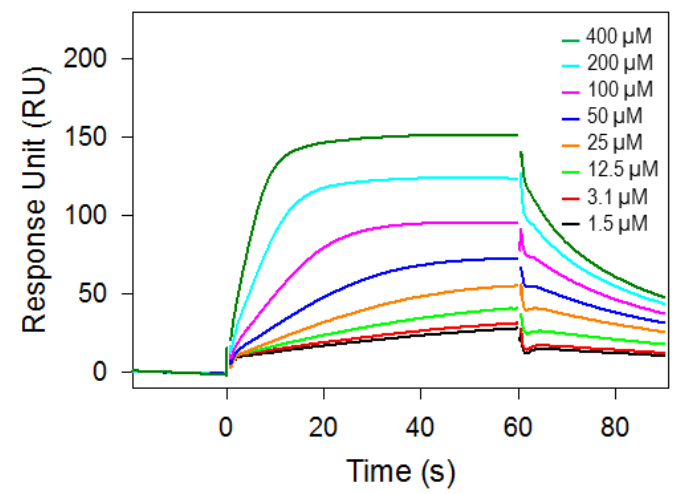

Figure 3. High-throughput screening (HTS) of DHOase. (A) Replicate plot of colorimetric enzymatic assay with fragments showing $>50 \%$ inhibition in red. (B) Replicate plot of thermal shift binding assay with fragments showing $>2{ }^{\circ} \mathrm{C}$ shift in red. (C) Melting curve of DHOase without substrate (black) and DHOase with Compound 4 (red). Compound 4 shows a shift in melting temperature $\left(\mathrm{T}_{\mathrm{m}}\right)$ as indicated by the arrow. (D) Sensorgram of Compound $\mathbf{4}$ at increasing concentrations. 

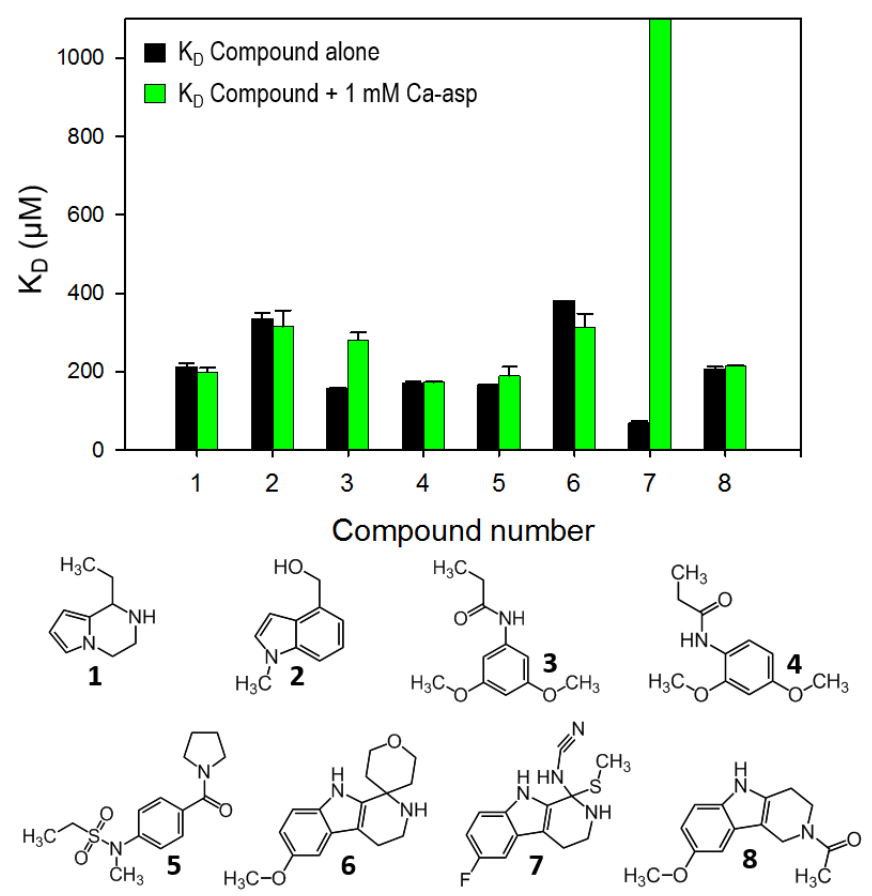

Figure 4. High-throughput screening hits. Bar graphs of the dissociation equilibrium constants $\left(\mathrm{K}_{\mathrm{D}}\right)$ of 8 initial hit compounds determined by Surface Plasmon Resonance $(\mathrm{SPR})$. $\mathrm{K}_{\mathrm{D}}$ values were compared in the presence (green) and in the absence (black) of the substrate Ca-asp as a competitor. Bars that reached the top of the graph represent $\mathrm{K}_{\mathrm{D}}$ values of over $1000 \mu \mathrm{M}$ (no binding).

\subsection{Identification of a lead compound and Structure-Activity Relationship (SAR)}

To determine if the inhibitors bind to the active site, the $\mathrm{K}_{\mathrm{D}}$ was determined for each compound in the absence and presence of substrate Ca-asp (Fig. 4). If the compounds bind to the active site, the expectation is that the compound $\mathrm{K}_{\mathrm{D}}$ should be affected by the presence of high concentration of substrate. Overall, the binding affinity did not change in the presence of $1 \mathrm{mM}$ Ca-asp, suggesting the hits may not be competitive inhibitors, with the exception of compound 7 (Fig. S7). These results suggest that the compounds may bind to an allosteric site, or possibly in the active site but not in a position to compete with substrate. Our crystal structure shows an 
open active site with a large chemical space which could accommodate two smaller fragments, such as our hit compounds and the natural substrate. This possibility could be observed when comparing compounds 3 and 4 . Compounds $\mathbf{3}$ and $\mathbf{4}$ are similar in structure with similar binding affinities of $157 \pm 2 \mu \mathrm{M}$ and $170 \pm 4 \mu \mathrm{M}$, respectively. However, with $1 \mathrm{mM} \mathrm{Ca}$-asp, the $\mathrm{K}_{\mathrm{D}}$ of compound $\mathbf{3}$ nearly doubled, to $281 \pm 19 \mu \mathrm{M}$, indicating that Ca-asp and $\mathbf{3}$ may be competing at least in part for the active site, whereas that of compound $\mathbf{4}$ remained the same. Previously, it has been shown that DHOase is highly specific for its substrate, and the substitution positions played a critical role, in that 5-substituted analogs of the substrate were more effective inhibitors and more competitive for the position in the active site that is normally occupied by either DHO or Ca-asp. ${ }^{27}$ Additionally, this may explain why compound $\mathbf{3}$ exhibited competition with the substrate, while compound 4 did not. Compounds 6-8 have a similar and much more rigid 3-ring scaffold, with compound 7 having the highest affinity for DHOase with a $K_{D}$ value of $67 \pm 7$ $\mu \mathrm{M}$. Interestingly, in the presence of substrate, the binding affinity weakened significantly enough that $\mathrm{K}_{\mathrm{D}}$ was unable to be determined, confirming that 7 is a competitive inhibitor.

A few commercially available fragment analogs of compounds $\mathbf{6}$ and $\mathbf{7}$ were obtained and tested by SPR, and a preliminary Structure-Activity-Relationships (SAR) is summarized in Table 3. When the tetrahydro- $2 H$-pyran ring of compound 6 was removed from $\mathrm{R}^{2}$ position, the $\mathrm{K}_{\mathrm{D}}$ increased $\sim 1.5$-fold, going from $380 \mu \mathrm{M}$ to $588 \mu \mathrm{M}$ and was not affected by the addition of substrate. When the methoxy group of $\mathbf{6 a}$ in the $\mathrm{R}^{1}$ position was replaced by a methyl group (6b), the compound interaction with DHOase was completely abolished. Similarly, when the methoxy group in the $\mathrm{R}^{1}$ was retained, but the tetrahydropyridine of $\mathrm{R}^{2}$ was removed $(\mathbf{6 c})$, the compound also showed no interaction with DHOase, indicating the interactions of the methoxy group and pyridine ring are necessary for active site binding. When the $\mathrm{R}^{1}$ position was kept as 
the same methoxy group and the pyran ring in $\mathrm{R}^{1}$ position was substituted by thiomethyl and cyanamide (7a), the binding affinity increased 18 -fold from $380 \mu \mathrm{M}$ to $21 \mu \mathrm{M}$, most likely due to the interaction between cyanamide and the $\mathrm{Zn}$ ions in the active site. When the methoxy group replaced the fluorine (7) in $\mathrm{R}^{1}$ position and kept $\mathrm{R}^{2}$ position same, the affinity decreased slightly to $84 \mu \mathrm{M}$. When the tetrahydropyridine ring was tethered with acetyl on the nitrogen (8) in the $\mathrm{R}^{2}$ position, interaction became weaker. These results together suggest that fluorine is the most favored, and methoxy is tolerated in the $\mathrm{R}^{1}$ position. A tetrahydropyridine in $\mathrm{R}^{2}$ is required, and being tethered with thiomethyl and cyanamide is the most favored, while tetrahydropyridine alone, fused with pyran, or tethered with acetyl on the nitrogen is tolerated (Fig.5). Based on this preliminary Structure-Activity Relationship (SAR) map, our lead compound, 7, can be further optimized through synthesis.

Table 3. Preliminary Structure-Activity Relationship (SAR) of HTS hit analogs.

\begin{tabular}{|c|c|c|c|c|c|}
\hline Compound & MW & $\mathrm{R}^{1}$ & $\mathrm{R}^{2}$ & $\mathrm{~K}_{\mathrm{D}}{ }^{\mathrm{a}}(\mu \mathrm{M})$ & $\begin{array}{l}\mathrm{K}_{\mathrm{D}}(\mu \mathrm{M}) \text { with } \\
1 \mathrm{mM} \text { Ca-asp }\end{array}$ \\
\hline 6 & 308.8 & $-\mathrm{OCH}_{3}$ & & $380 \pm 1$ & $314 \pm 34$ \\
\hline $6 \mathbf{a}$ & 202.3 & $-\mathrm{OCH}_{3}$ & & $588 \pm 38$ & $675 \pm 12$ \\
\hline $6 b$ & 186.3 & $-\mathrm{CH}_{3}$ & & $\mathrm{NB}^{\mathrm{b}}$ & $\mathrm{NB}^{\mathrm{b}}$ \\
\hline 6c & 176.2 & $-\mathrm{OCH}_{3}$ & $-2-\mathrm{CH}_{2} \mathrm{NH}_{2}$ & $\mathrm{NB}^{\mathrm{b}}$ & $\mathrm{NT}^{\mathrm{c}}$ \\
\hline 7 & 276.3 & $-F$ & & $67 \pm 7$ & $>1 \mathrm{mM}$ \\
\hline
\end{tabular}


$7 \mathbf{a}$ 288.4 $-\mathrm{OCH}_{3}$<smiles>CSC1(NC=N)C=CCCN1</smiles>
$119 \pm 13$ $144 \pm 69$

8 244.3 $-\mathrm{OCH}_{3}$

$$
205 \pm 8
$$

${ }^{\mathrm{a}} \mathrm{K}_{\mathrm{D}}$ : Equilibrium dissociation constant determined by SPR; the standard deviation are from three independent experiments.

b NB: Non-specific binding pattern

c: NT: Not tested
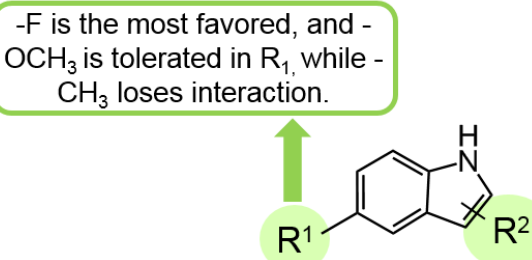

Tetrahydropyridine tethered with thiomethyl and cyanamide is the most favored in $\mathrm{R}_{2}$. Tetrahydropyridine alone, fused with pyran, or tethered with acetyl on nitrogen are tolerated.

Figure 5. Preliminary Structure-Activity Relationship (SAR) map of HTS hit analogs. The $\mathrm{K}_{\mathrm{D}}$ of nine similarly structured fragment compounds were analyzed to generate this map.

\section{Conclusions}

We were able to solve the crystal structure of $B$. anthracis DHOase with Ca-asp in the active site (PDB: 4YIW). The structure confirms that the key interactions between the substrate and active site residues are similar between Class I and Class II DHOase enzymes, except for the lack of substrate stabilization by the catalytic loop in Class I DHOase. The crystal structure of E. coli (PDB:1XGE) shows two threonine residues that stabilize the substrate, which may also play a role in stabilizing small inhibitors. In contrast, the shorter loop in B. anthracis DHOase interaction with the substrate is minimal, and our structure confirms the previously hypothesized role of glycine as the hydrogen bond contributor. To further understand the importance of Caasp stabilization by hydrogen bonding, the enzyme kinetics will be compared to G152S and 
G152T in ongoing studies. The lack of loop stabilization may be one of the reasons why inhibitors of Class II DHOase have not been effective in B. anthracis DHOase. In order to identify potential inhibitors, we performed high-throughput screening of B. anthracis DHOase against several libraries using an enzymatic assay and an orthogonal binding assay. The binding affinity constants for eight inhibitors were determined by SPR, as well as by competition analysis with Ca-asp to determine if the inhibitors bound in the active site. The few hit compounds share a similar scaffold, and analogs were selected for further analysis based on binding affinities, with a preliminary SAR map. Our results provide more insight into the dihydroorotase family and identified several compounds that can be further optimized as potential antimicrobials against $B$. anthracis.

\section{Experimental section}

\subsection{Preparation and purification of DHOase}

The DHOase gene from Bacillus anthracis (Sterne strain) was cloned into a pET15b vector

(Invitrogen) and purified as previously described. ${ }^{18,28}$ In brief, the recombinant plasmid was inserted into BL21(DE3) Gold cells (Invitrogen) and grown in Terrific Broth (TB) media (Fisher Scientific) at $37^{\circ} \mathrm{C}$ while shaking at $220 \mathrm{rpm}$ until the $\mathrm{OD}_{600}$ reached 0.6 , when it was induced with $1 \mathrm{mM}$ isopropyl B-D-1-thiogalactopyranoside (IPTG) (Sigma) and incubated for an additional 4 hours before harvesting. The cell pellet was resuspended in $50 \mathrm{mM}$ Tris, $\mathrm{pH} 8.0$, $500 \mathrm{mM} \mathrm{NaCl}, 20 \mathrm{mM}$ imidazole, and $5 \mathrm{mM} \beta$-mercaptoethanol ( $\beta$-MCE), $1 \mathrm{mg} / \mathrm{mL}$ lysozyme, $0.01 \%$ Triton X-100, and $0.025 \mathrm{mg} / \mathrm{mL}$ DNase I, and lysed by sonication. A HisTrap HP column (GE Healthcare) was used to purify the histidine-tagged protein using a stepwise 
gradient of elution buffer ( $50 \mathrm{mM}$ Tris, $\mathrm{pH} 8.0,500 \mathrm{mM} \mathrm{NaCl}, 500 \mathrm{mM}$ imidazole, and $5 \mathrm{mM} \beta$ MCE) with either an AKTA purifier or AKTAxpress FPLC system. The eluted protein was dialyzed in $50 \mathrm{mM}$ Tris, $\mathrm{pH} 7.5$, and $100 \mathrm{mM} \mathrm{NaCl}$ overnight to remove imidazole. The histidine tag was removed by incubating the dialyzed protein with 1.5 units of thrombin per $\mathrm{mg}$ protein at room temperature for 1 hour, followed by 45 minutes at $37^{\circ} \mathrm{C}$. The digested protein was reloaded onto a HisTrap HP column stacked with a HiTrap Benzamidine column that was equilibrated with $50 \mathrm{mM}$ Tris, $\mathrm{pH} 8.0,500 \mathrm{mM} \mathrm{NaCl}$, and $5 \mathrm{mM} \beta$-MCE. The histidine-tag cleaved DHOase was collected in the flow-through and loaded onto a HiLoad 16/60 Superdex 75 PG gel filtration column that was equilibrated with $25 \mathrm{mM}$ Tris, $\mathrm{pH} 7.5$ and $100 \mathrm{mM} \mathrm{NaCl}$. Protein samples were analyzed by SDS-PAGE and final purity was above $90 \%$.

\subsection{Site-directed mutagenesis}

R63A, N93A, and D304A mutations on DHOase were generated by site-directed mutagenesis PCR using the NEB Q5 mutagenesis Kit and wild-type pET15b plasmid containing $B$. anthracis DHOase as a template. The primers used are as follows: R63A, 5'-

GGATTAGTAGATGTACACGTACACCT GCAGAACCAGGTGGTGAACATAAAG-3'; N93A, 5'-CACTACAATTTGCGCAATGCCAGCAACACGCCCAGTACCAG-3'; D304A, 5'GGAACAATCGATATGATCGCAACTGCACATGCACCGCATACAGC-3'. The PCR reaction contained $0.3 \mu \mathrm{M}$ each of forward and reverse primers, $0.5 \mu \mathrm{L}$ template, $12.5 \mu \mathrm{L}$ Q5 Hot Start High-Fidelity $2 \mathrm{X}$ Mix, and sterile water adjusted to $50 \mu \mathrm{L}$ total volume for each forward and reverse reaction. The PCR product was incubated with the provided Kinase-LigaseDpnI enzyme mix for 30 minutes at room temperature. The final PCR product was transformed into DH5 $\alpha$ cells via heat shock for 30 seconds at $42^{\circ} \mathrm{C}$ and plated onto ampicillin treated plates (ampicillin $100 \mu \mathrm{g} / \mathrm{mL}$ ). Transformants with the correct mutation were used to extract the 
plasmid and retransform into a protein expression cell line, BL21(DE3) Gold cells, via heat shock at $42^{\circ} \mathrm{C}$ for 30 seconds. Each mutant was expressed and purified by the same way as the wild-type DHOase.

\subsection{Enzyme kinetics determination}

The Michaelis-Menten constant $\left(\mathrm{K}_{\mathrm{M}}\right)$ was determined as described previously. ${ }^{18,25}$ In brief, the $\mathrm{K}_{\mathrm{m}}$ values were determined by monitoring the UV absorbance at $230 \mathrm{~nm}$. The reverse reaction of $100 \mathrm{nM}$ final concentration wild-type DHOase or its mutants (R63A, N93A, or D304ADHOase) were performed in $50 \mathrm{mM}$ Tris, $\mathrm{pH} 8.3$ with concentrations of substrate DHO ranging from $0-500 \mu \mathrm{M}$ at 2-fold serial dilution in a 96-well UV plate (Corning). The forward reaction was performed in the same manner, but with a different assay buffer consisting of $50 \mathrm{mM}$ MES, pH 5.8 at $100 \mathrm{nM}$ final concentration wild-type DHOase or its mutants. The substrate Ca-asp concentrations for wild-type and two mutants (R63A and D304A) DHOase reaction ranged from $0-650 \mu \mathrm{M}$, while a much higher concentration range $(0-5 \mathrm{mM})$ was used for N93A-DHOase. The enzyme reaction was continuously monitored using a SPECTRAmax Plus (Molecular Devices). The $\mathrm{K}_{\mathrm{M}}$ value and maximum activity $\left(\mathrm{V}_{\max }\right)$ were calculated using Sigmaplot 11.0 to fit the data to the hyperbolic equation (1), where $\mathrm{y}$ is the initial velocity and $\mathrm{x}$ is the concentration of the substrate.

\subsection{Crystallization of substrate-bound BaDHOase}

The pooled histidine-tag cleaved wild-type DHOase was concentrated to $10 \mathrm{mg} / \mathrm{mL}$. It was then incubated with $1.5 \mathrm{mM}$ of either DHO or Ca-asp overnight on ice. DHOase was crystallized using hanging-drop vapor diffusion with the crystallization condition of 0.1 M Bis-Tris, pH 5.5, $0.2 \mathrm{M} \mathrm{NaCl}$, and 20\% PEG 3350. The crystals diffracted at $\lambda=0.979800 \AA$ at $100 \mathrm{~K}$ using a MARMOSAIC CCD $300 \mathrm{~mm}$ detector at an oscillation angle of $1.0^{\circ}$ for 360 frames at the 
Advanced Photon Source LS-cat beamline, station 21-ID-D at the Argonne National Laboratory. The data was indexed, integrated, and scaled by XDS. ${ }^{29}$ The molecular replacement was completed using Phaser ${ }^{30}$ in $\mathrm{CCP} 4$ and the structural refinement was completed with Refmac5.5 $5^{31}$ and $\operatorname{Coot}^{32}$.

\subsection{Primary high-throughput screening}

High-throughput screening of 3,352 fragments from the Chembridge fragment and Zenobia fragment libraries were performed using the Tecan Freedom EVO 200 liquid handling robot and the DAMO-TSC assay which has previously been optimized for this target. ${ }^{18}$ All assays were done in duplicate in transparent 384-well plates (Grenier). $30 \mu \mathrm{L}$ of $133 \mathrm{nM}$ his-tagged DHOase in HTS buffer (50 mM Tris, pH 8.3, 0.01\% Triton X-100, $0.1 \mathrm{mg} / \mathrm{mL}$ BSA, 4 mM TCEP) was dispensed to plates, and final concentrations of $400 \mu \mathrm{M}$ of each fragment or $25 \mu \mathrm{M}$ of each compound was added and incubated for 10 minutes at room temperature. Then $10 \mu \mathrm{L}$ of $320 \mu \mathrm{M}$ substrate DHO in the same assay buffer was added prior to incubation for another 30 minutes after 30 seconds of shaking. To quench the reaction, $64 \mu \mathrm{L}$ of DAMO-TSC acid color mix was added. The assay plates were sealed and incubated in the dark for 16 hours at room temperature prior to measuring the absorbance at $540 \mathrm{~nm}$. Each plate contained 32 positive and 32 negative controls.

\subsection{Fluorescence thermal shift assay}

A fluorescence thermal shift (FTS) binding assay was performed on 3,352 fragments. FTS assay plates were prepared by the Tecan Freedom EVO 200 liquid handling robot, and melting curves were monitored using a Viia7 Real-Time PCR (Applied Biosystems). $5 \mu \mathrm{L}$ of histidine-tagged DHOase (5 $\mu \mathrm{M}$ final concentration) in FTS buffer consisting of $50 \mathrm{mM}$ MES, pH 6.5, 0.01\% 
CHAPS, $150 \mathrm{mM} \mathrm{NaCl}$, and $2 \mathrm{mM}$ TCEP was dispensed in white 384-well PCR plates

(BioRad). $0.1 \mu \mathrm{L}$ of each fragment (400 $\mu \mathrm{M}$ final concentration) was added to the plate using a 384-pin stainless steel pin tool (V\&P Scientific) with a 100-nL capillary capacity and incubated for 10 minutes at room temperature. Then $5 \mu \mathrm{L}$ of 10X SYPRO Orange dye, diluted from 5000X stock, was added prior to shaking for 30 seconds. The assay plates were gradually heated from $25^{\circ} \mathrm{C}$ to $95^{\circ} \mathrm{C}$ at $0.07{ }^{\circ} \mathrm{C} /$ min while the fluorescence intensity was continuously measured at excitation/emission 470/623 nm. A Protein Thermal Shift ${ }^{\mathrm{TM}}$ Software (Applied Biosystems) was used to calculate the $T_{m}$ shifts, and any fragments with $T_{m}>2^{\circ} \mathrm{C}$ were identified as positive hits.

\subsection{Hit validation with surface plasmon resonance}

Purified histidine-tag cleaved DHOase was buffer exchanged to PBS-P (10 mM phosphate, $\mathrm{pH}$ 7.4, $2.7 \mathrm{mM} \mathrm{KCl}, 137 \mathrm{mM} \mathrm{NaCl}$, and 0.05\% surfactant P-20). Surface Plasmon Resonance was performed at $25{ }^{\circ} \mathrm{C}$ using a Biacore T200 instrument and a CM5 sensor chip. The flow channels were activated by a 1-ethyl-3-(3-dimethylaminopropyl) carbodiimide hydrocholoride (EDC)/ Nhydroxy succinimide (NHS) mixture. DHOase was diluted in $10 \mathrm{mM}$ sodium acetate (pH 4.5) and immobilized to flow channels 2, 3, and 4 at levels of 12,974 RU, 9,853 RU, and 12,150 RU, respectively. Flow channel 1 was blocked by ethanolamine ( $\mathrm{pH} 8.5)$ as a control. Compound solutions at increasing concentrations (0-200 $\mu \mathrm{M}$ at 2-fold dilutions) in binding buffer were applied to all four channels at a $10 \mu \mathrm{L} / \mathrm{min}$ flow rate. The SPR binding buffer was PBS-P, $2 \%$ DMSO and $0.5 \mathrm{mM}$ TCEP. $1 \mathrm{mM}$ Ca-asp was added to the binding buffer for competition SPR. The sensorgrams were analyzed using Biaevaluation software 2.0.3. All data was reference with the blank channel response unit signal prior to the fitting. The response unit difference at each concentration was measured during the binding equilibration phase and $K_{D}$ values were 
determined using the steady-state affinity fitting equation embedded in the Biaevaluation software.

\section{Acknowledgements}

AJR was supported during a portion of this work by NIDCR T32-DE018381, UIC College of Dentistry, MOST Program. We thank Dr. Kiira Ratia for compound library preparation and preparation of TSA plates, and Dr. Hyunwoo Lee for the B. anthracis $\triangle$ ANR strain. This research used resources of the Advanced Photon Source, a U.S. Department of Energy (DOE) Office of Science User Facility operated for the DOE Office of Science by Argonne National Laboratory under Contract No. DE-AC02-06CH11357. Use of the LS-CAT Sector 21 was supported by the Michigan Economic Development Corporation and the Michigan Technology Tri-Corridor (Grant 085P1000817). Molecular graphics and analyses were performed with the UCSF Chimera package. Chimera was developed by the Resource for Biocomputing, Visualization, and Informatics at the University of California, San Francisco (supported by NIGMS P41-GM103311). 


\section{References}

1. D.R. Evans, H.I. Guy, Mammalian pyrimidine biosynthesis: fresh insights into an ancient pathway, J Biol Chem, 279 (2004) 33035-33038.

2. T.N. Porter, Y. Li, F.M. Raushel, Mechanism of the dihydroorotase reaction, Biochemistry, 43 (2004) 16285-16292.

3. A. Grande-García, N. Lallous, C. Díaz-Tejada, S. Ramón-Maiques, Structure, functional characterization, and evolution of the dihydroorotase domain of human CAD, Structure, 22 (2014) 185-198.

4. L. Holm, C. Sander, An evolutionary treasure: unification of a broad set of amidohydrolases related to urease, Proteins, 28 (1997) 72-82.

5. A. Liu, L. Huo, Amidohydrolase Superfamily, eLS, John Wiley \& Sons, Ltd2001.

6. E.G. Sander, M.J. Heeb, Purification and properties of dihydroorotase from Escherichia coli B, Biochim Biophys Acta, 227 (1971) 442-452.

7. C.C. Wang, H.W. Tsau, W.T. Chen, C.Y. Huang, Identification and characterization of a putative dihydroorotase, KPN01074, from Klebsiella pneumoniae, Protein J, 29 (2010) 445452.

8. M. Lee, C.W. Chan, J. Mitchell Guss, R.I. Christopherson, M.J. Maher, Dihydroorotase from Escherichia coli: loop movement and cooperativity between subunits, J Mol Biol, 348 (2005) 523-533.

9. M. Lee, C.W. Chan, S.C. Graham, R.I. Christopherson, J.M. Guss, M.J. Maher, Structures of ligand-free and inhibitor complexes of dihydroorotase from Escherichia coli: implications for loop movement in inhibitor design, J Mol Biol, 370 (2007) 812-825.

10. M. Lee, M.J. Maher, R.I. Christopherson, J.M. Guss, Kinetic and structural analysis of mutant Escherichia coli dihydroorotases: a flexible loop stabilizes the transition state, Biochemistry, 46 (2007) 10538-10550.

11. M. Lee, M.J. Maher, J.M. Guss, Structure of the T109S mutant of Escherichia coli dihydroorotase complexed with the inhibitor 5-fluoroorotate: catalytic activity is reflected by the crystal form, Acta Crystallogr Sect F Struct Biol Cryst Commun, 63 (2007) 154-161.

12. J.B. Thoden, G.N. Phillips, Jr., T.M. Neal, F.M. Raushel, H.M. Holden, Molecular structure of dihydroorotase: a paradigm for catalysis through the use of a binuclear metal center, Biochemistry, 40 (2001) 6989-6997.

13. S. Samant, H. Lee, M. Ghassemi, J. Chen, J.L. Cook, A.S. Mankin, A.A. Neyfakh, Nucleotide biosynthesis is critical for growth of bacteria in human blood, PLoS Pathog, 4 (2008) e37.

14. T. Berger, M. Kassirer, A.A. Aran, Injectional anthrax - new presentation of an old disease, Euro Surveill, 19 (2014).

15. D.A. Sweeney, C.W. Hicks, X. Cui, Y. Li, P.Q. Eichacker, Anthrax infection, Am J Respir Crit Care Med, 184 (2011) 1333-1341.

16. W.M. Hendricks KA, Shadomy SV, Bradley JS, Morrow MG, Pavia AT, et al., Centers for Disease Control and Prevention expert panel meetings on prevention and treatment of anthrax in adults, Emerg Infect Dis [Internet], (2014).

17. J.L. Owen, T. Yang, M. Mohamadzadeh, New insights into gastrointestinal anthrax infection, Trends Mol Med, 21 (2015) 154-163. 
18. A.J. Rice, L. Truong, M.E. Johnson, H. Lee, A colorimetric assay optimization for highthroughput screening of dihydroorotase by detecting ureido groups, Anal Biochem, 441 (2013) 87-94.

19. M. Knipp, M. Vasak, A colorimetric 96-well microtiter plate assay for the determination of enzymatically formed citrulline, Analytical Biochemistry, 286 (2000) 257-264.

20. F.J. Sorrell, G.K. Greenwood, K. Birchall, B. Chen, Development of a differential scanning fluorimetry based high throughput screening assay for the discovery of affinity binders against an anthrax protein, J Pharm Biomed Anal, 52 (2010) 802-808.

21. M.C. Lo, A. Aulabaugh, G. Jin, R. Cowling, J. Bard, M. Malamas, G. Ellestad, Evaluation of fluorescence-based thermal shift assays for hit identification in drug discovery, Anal Biochem, 332 (2004) 153-159.

22. C.J. Layton, H.W. Hellinga, Thermodynamic analysis of ligand-induced changes in protein thermal unfolding applied to high-throughput determination of ligand affinities with extrinsic fluorescent dyes, Biochemistry, 49 (2010) 10831-10841.

23. M.W. Pantoliano, E.C. Petrella, J.D. Kwasnoski, V.S. Lobanov, J. Myslik, E. Graf, T. Carver, E. Asel, B.A. Springer, P. Lane, F.R. Salemme, High-density miniaturized thermal shift assays as a general strategy for drug discovery, J Biomol Screen, 6 (2001) 429-440.

24. R. Karlsson, Real-time competitive kinetic analysis of interactions between lowmolecular-weight ligands in solution and surface-immobilized receptors, Anal Biochem, 221 (1994) 142-151.

25. L. Truong, K.E. Hevener, A.J. Rice, K. Patel, M.E. Johnson, H. Lee, High-level expression, purification, and characterization of Staphylococcus aureus dihydroorotase (PyrC) as a cleavable His-SUMO fusion, Protein Expr Purif, 88 (2013) 98-106.

26. G. Andreotti, M. Monticelli, M.V. Cubellis, Looking for protein stabilizing drugs with thermal shift assay, Drug Test Anal, (2015).

27. R.I. Christopherson, M.E. Jones, The effects of $\mathrm{pH}$ and inhibitors upon the catalytic activity of the dihydroorotase of multienzymatic protein pyr1-3 from mouse Ehrlich ascites carcinoma, J Biol Chem, 255 (1980) 3358-3370.

28. S. Mehboob, D.C. Mulhearn, K. Truong, M.E. Johnson, B.D. Santarsiero, Structure of dihydroorotase from Bacillus anthracis at 2.6 ̊ resolution, Acta Crystallogr Sect F Struct Biol Cryst Commun, 66 (2010) 1432-1435.

29. W. Kabsch, XDS, Acta Crystallogr D Biol Crystallogr, 66 (2010) 125-132.

30. A.J. McCoy, R.W. Grosse-Kunstleve, P.D. Adams, M.D. Winn, L.C. Storoni, R.J. Read, Phaser crystallographic software, J Appl Crystallogr, 40 (2007) 658-674.

31. A.A. Vagin, R.A. Steiner, A.A. Lebedev, L. Potterton, S. McNicholas, F. Long, G.N. Murshudov, REFMAC5 dictionary: organization of prior chemical knowledge and guidelines for its use, Acta Crystallogr D Biol Crystallogr, 60 (2004) 2184-2195.

32. P. Emsley, B. Lohkamp, W.G. Scott, K. Cowtan, Features and development of Coot, Acta Crystallogr D Biol Crystallogr, 66 (2010) 486-501. 


\section{Figure Legends}

Figure 1. The mechanism of DHOase. (A) The enzyme catalyzes the reversible cyclization of Ca-asp to DHO. (B) Ca-asp is stabilized by the $\mathrm{Zn}^{2+}$ ions coordinated by a carboxylated $\mathrm{K} 102$, while D250 abstracts the proton from the amide nitrogen of Ca-asp. (PDB: 1XGE). The exocyclic carboxyl group of Ca-asp is stabilized by N44, R20, and H254, in addition to the T109 and T110 from the 'in' formation of the catalytic loop. (C) Comparison of the three classes of DHOase with the catalytic loop highlighted in pink.

Figure 2. Comparison of Class I and Class II DHOase. (A) Electron density of Ca-asp in the active site. The ligand density is countered at $2 \mathrm{~F}_{\mathrm{o}}-\mathrm{F}_{\mathrm{c}}=0.51 \mathrm{rmsd}$. (B) Overlay of substrate bound B. anthracis DHOase (PDB:4YIW, cyan) and E. coli DHOase (PDB:1XGE, tan). (C) Catalytic loop of E. coli DHOase (PDB:1XGE) highlighted in blue, and H-bond interaction of substrate with the peptide backbone of glycine in $B$. anthracis DHOase. (PDB:4YIW). (D) Overlay of the catalytic loop. (B. anthracis apo PDB:3MPG; B. anthracis + Ca-asp PDB:4YIW; E. coli apo PDB:2EG6; E. coli + Ca-asp PDB:1XGE; human apo PDB:4BYE; human + Ca-asp PDB:4C6I). (E) Sequence alignment of catalytic loop highlighted in yellow.

Figure 3. High-throughput screening (HTS) of DHOase. (A) Replicate plot of colorimetric enzymatic assay with fragments showing $>50 \%$ inhibition in red. (B) Replicate plot of thermal shift binding assay with fragments showing $>2{ }^{\circ} \mathrm{C}$ shift in red. (C) Melting curve of DHOase without substrate (black) and DHOase with Compound 4 (red). Compound 4 shows a shift in melting temperature $\left(\mathrm{T}_{\mathrm{m}}\right)$ as indicated by the arrow. (D) Sensorgram of Compound $\mathbf{4}$ at increasing concentrations.

Figure 4. High-throughput screening hits. Bar graphs of the dissociation equilibrium constants $\left(\mathrm{K}_{\mathrm{D}}\right)$ of 8 initial hit compounds determined by Surface Plasmon Resonance $(\mathrm{SPR})$. $\mathrm{K}_{\mathrm{D}}$ 
values were compared in the presence (green) and in the absence (black) of the substrate Ca-asp as a competitor. Bars that reached the top of the graph represent $K_{D}$ values of over $1000 \mu \mathrm{M}$ (no binding).

Figure 5. Preliminary Structure-Activity Relationship (SAR) map of HTS hit analogs. The $\mathrm{K}_{\mathrm{D}}$ of nine similarly structured fragment compounds were analyzed to generate this map. 


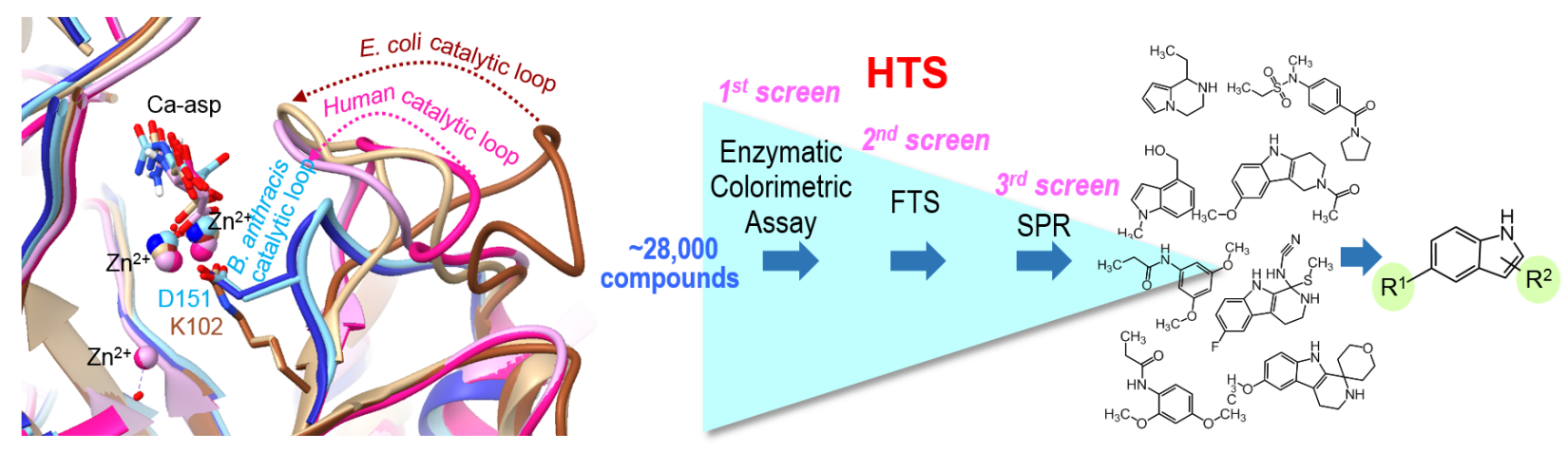

